\title{
Why there are two cyclooxygenase isozymes
}

\author{
William L. Smith ${ }^{1}$ and Robert Langenbach ${ }^{2}$ \\ ${ }^{1}$ Department of Biochemistry and Molecular Biology, Michigan State University, East Lansing, Michigan, USA \\ ${ }^{2}$ Laboratory of Environmental Carcinogenesis and Mutagenesis, National Institute of Environmental Health Sciences, \\ Research Triangle Park, North Carolina, USA \\ Address correspondence to: William L. Smith, 513 Biochemistry Building, Department of Biochemistry and Molecular Biology, \\ Michigan State University, East Lansing, Michigan 48824, USA. \\ Phone: (517) 355-1604; Fax: (517) 353-9334; E-mail: smithww@msu.edu.
}

Since the discovery in 1991 of a second isoform of prostaglandin endoperoxide $\mathrm{H}$ synthase (PGHS, or cyclooxygenase), there has been considerable interest in the question of why two isoforms of this enzyme are necessary and what roles they might play. PGHS-1deficient (1) and PGHS-2-deficient $(2,3)$ mice and isoform-specific inhibitors have been developed and used to investigate the physiological functions of PGHS-1 and PGHS-2. These studies suggest that there are processes in which each isozyme is uniquely involved (e.g., platelet aggregation for PGHS-1, ovulation for PGHS-2) and others in which both isozymes function coordinately (e.g., carcinogenesis, inflammation). There are also physiological events in which one PGHS isozyme normally functions but for which the other can compensate when the first is lacking (e.g., parturition and remodeling of the ductus arteriosus). Biochemical studies indicate that each isoform can function independently; namely, that there are distinct PGHS-1 and PGHS-2 prostanoid biosynthetic pathways. Thus, the unique physiological roles for each isozyme can be rationalized by what is known about the biochemistry of the enzymes.

To facilitate discussion of the physiological functions of PGHS- 1 and PGHS- 2 and to point out those functions for which PGHS-1 and PGHS- 2 can substitute for one another, we describe the following in sequence: (a) physiological processes that depend solely or primarily on PGHS-1, (b) physiological processes that depend solely or primarily on PGHS-2, and (c) processes in which both PGHS-1 and PGHS-2 are involved and act coordinately. We then summarize the biochemical evidence for distinct PGHS-1 and PGHS-2 biosynthetic pathways.

\section{Physiological processes for which}

PGHS-1 is responsible

Platelet aggregation. Mature platelets are essentially nonnucleated cellular vesicles that mediate hemostasis by aggregating in response to arterial injury. Platelets can form thromboxane $\mathrm{A}_{2}\left(\mathrm{TXA}_{2}\right)$ from arachidonic acid (AA) supplied either exogenously or generated from endogenous phospholipid stores (see Brash, this Perspective series, ref. 4). TXA 2 exits the cells and acts via the platelet $\mathrm{TXA}_{2}$ receptor (TP) to initiate platelet shape changes and subsequent platelet aggregation (5). $\mathrm{TXA}_{2}$ can also act via TP receptors to cause constriction of vascular smooth muscle (5). Platelets express PGHS-1 but not PGHS-2. Thus, $\mathrm{TXA}_{2}$ is formed by platelets only via PGHS-1 (see Table 1) (6). Not surprisingly, platelets from PGHS-1-null mice fail to undergo AA-induced aggregation (1), indicating that PGHS-2 cannot compensate for an absence of PGHS-1 in platelets. Low-dose aspirin, used therapeutically as an anti-thrombogenic agent, targets platelet PGHS-1 (7).

Parturition. The PGHS isozymes play distinct physiological roles in female reproduction. Early studies demonstrated that PGHS-1-null female mice produced litters of normal size but had difficulty with parturition (1), whereas other aspects of the reproductive process in PGHS-1-null mice were normal (8). Gross et al. (9) also found that the onset of parturition was delayed in PGHS-1-null mice and observed that administration of $\mathrm{PGF}_{2 \alpha}$ resulted in the onset of labor, suggesting that PGHS-1 is the source of the PGF $2 \alpha$ (Table 1). However, treatment with LPS, a bacterial component that induces cellular responses associated with septicemia, induces PGHS-2 and allows parturition to proceed, even in PGHS-1-null mice. Use of SC236, a PGHS-2-selective nonsteroidal anti-inflammatory drug (NSAID), blocks the onset of parturition in these LPS-treated mice (10). Thus, under certain pathological conditions, $\mathrm{PGHS}-2$ can also produce the $\mathrm{PGF}_{2 \alpha}$ needed to initiate parturition.

\section{Physiological processes for which}

PGHS-2 is responsible

Ovulation and implantation. PGHS-2-null female mice are infertile (3). Although follicular development is observed in PGHS-2-deficient mice, ovulation is greatly reduced and, of the eggs released, very few are fertilized (8). Ovarian $\mathrm{PGE}_{2}$ production is increased by pituitary gonadotropins in wild-type and PGHS-1-null mice, but not in PGHS-2-null mice, indicating that PGHS-2 is responsible for this increased ovarian $\mathrm{PGE}_{2}$ production. Because ovulation in PGHS-2-null mice can be restored by $\mathrm{PGE}_{2}$ administration (11), $\mathrm{PGE}_{2}$ formed via PGHS-2 is believed to be the product that is responsible for ovulation. In addition to affecting ovu- 


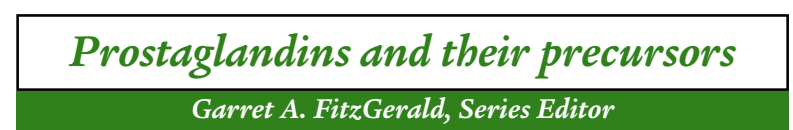

lation, PGHS-2 deficiency also impedes blastocyst implantation and decidualization. $\mathrm{PGI}_{2}$ has been demonstrated to be the prostanoid involved in this process, and it is also formed through PGHS-2 (Table 1) (12). Interestingly, although prostanoids appear to act primarily via $G$ protein-linked receptors (5), the nuclear peroxisomal proliferator-activated receptor PPAR $\delta$ appears to mediate the action of $\mathrm{PGI}_{2}$ in implantation (12), suggesting that alternative routes may exist by which $\mathrm{PGI}_{2}$ can influence gene expression.

Neonatal development. Although it is widely believed that PGHS-1 acts in development, there are few reports available to support this claim, and recent studies suggest that PGHS-2 plays a more important role, at least in neonatal development $(13,14)$. PGHS-2-deficient mice develop a severe renal pathology that is not mimicked by administration of NSAIDs to adult mice $(2,3)$. Initially, some investigators believed that this phenotype represented a compensatory artifact of the PGHS-2 knockout, but Komhoff et al. (13) showed recently that postnatal treatment with a PGHS-2-selective NSAID caused a severe reduction in glomerular diameter in the neonatal mouse kidney. This was the same renal pathology as seen in PGHS-2-null mice (13) and could not be caused by treating adult mice with the PGHS-2-selective inhibitor.

Another neonatal event in the mouse in which PGHS-2 has a key role is in the closure of the ductus arteriosus (14). Although the ductus closes normally in PGHS-1-null mice, about $35 \%$ of PGHS-2-null mice die with a patent ductus within 48 hours of birth. In wild-type mice, PGHS-2, but not PGHS-1, is seen by immunohistochemistry to be significantly induced in the smooth muscle cells of the ductus during closure; however, the fact that $65 \%$ of COX-2-null mice survived to weaning suggests that PGHS- 1 can play a compensatory role. Indeed, a reduction in PGHS-1 gene dosage from wild-type to hetrozygosity further increas- es the incidence of patent ductus arteriosus and decreases the 48-hour survival of PGHS-2-null mice to about $20 \%$. Because a role for PGHS-1 is only evident when PGHS- 2 is absent, it is unlikely that PGHS- 1 is involved in ductus closure in wild-type mice.

These examples indicate that PGHS-2 has key roles during postpartum development. Because the birthing process involves a number of physiological changes and stresses that could induce PGHS-2, and because PGHS-2-null mice have decreased survival at all ages, it may be useful to search for other neonatal tissues in which PGHS-2 induction promotes normal neonatal development.

Processes involving both PGHS-1 and PGHS-2 Inflammation and wound healing. The relative contributions of PGHS-1 and PGHS-2 to inflammatory responses are incompletely resolved. Since the identification of the PGHS-2 isoform, the hypothesis has been that PGHS-2 is the primary source of prostanoids that contribute to inflammation. However, several recent studies show that prostanoids formed via PGHS-1 are also involved $(1,15)$.

Interestingly, some recent studies have indicated that PGHS-2 acts in both the initiation of the inflammatory response and in the resolution phase. Gilroy et al. (16) recently reported that PGHS-2 expression and $\mathrm{PGE}_{2}$ levels increased transiently early in the course of carrageenan-induced pleurisy in rats. Later in the response, PGHS-2 was induced again to even greater levels and generated anti-inflammatory prostaglandins, such as $\mathrm{PGD}_{2}$ and 15 -deoxy- $\Delta^{12-14}-\mathrm{PGJ}_{2}$, but only low levels of the proinflammatory $\mathrm{PGE}_{2}$ (Table 1). Further support for an anti-inflammatory role of PGHS- 2 in this model was the finding that late administration of the PGHS-2-selective inhibitor NS-398 exacerbates the inflammatory response. Furthermore, Wallace et al. (15) observed that in the paw carrageenan inflammation model, the

\section{Table 1}

Isoform-specific physiological functions of PGHS-1 or PGHS-2

\begin{tabular}{|c|c|c|c|}
\hline Physiological process & PGHS-1 & PGHS-2 & $\begin{array}{l}\text { Prostaglandin } \\
\text { involved }\end{array}$ \\
\hline Ovulation & Not essential & Essential $(8,11)$ & $\mathrm{PGE}_{2}$ \\
\hline Implantation & Not essential & Essential (8) & $\mathrm{PGI}_{2}$ \\
\hline Parturition & Essential $(1,9)$ & Compensatory $(10)$ & $\mathrm{PGF}_{2 \alpha}$ \\
\hline Inflammation resolution & Not essential & Essential (16) & $\mathrm{PGD}_{2}, 15$-deoxy-PGJ ${ }_{2}$ \\
\hline Platelet aggregation & Essential (1) & No role & $\mathrm{TXA}_{2}$ \\
\hline Perinatal kidney development & Not essential (1) & Essential (13) & Not determined \\
\hline Ductus arteriosus remodeling & Compensatory & Essential (14) & $\mathrm{TXA}_{2} / \mathrm{PGH}_{2}$ \\
\hline T cell development & Stage-specific & Stage-specific (37) & $\mathrm{PGE}_{2}$ \\
\hline Gastric ulceration & \multicolumn{2}{|c|}{ Inhibition of both isoforms necessary (17) } & Not determined \\
\hline Ulcer healing & Not essential & Essential $(15,18)$ & Not determined \\
\hline Intestinal cancer & \multicolumn{2}{|c|}{ Both isoforms have essential roles (20) } & Not determined \\
\hline Crypt stem cell survival & Essential (21) & Compensatory (38) & $\mathrm{PGE}_{2}$ \\
\hline
\end{tabular}


inflammation resolves within 7 days in wild-type mice but is unmitigated over this period in PGHS-2-deficient mice. Thus, PGHS-2 appears to have two roles in the inflammatory process, initially contributing to the onset of inflammation and later helping to resolve the process.

Gastric ulceration. The inhibition of PGHS-1 by NSAIDs has for many years been considered the biochemical event responsible for gastric ulcer development. It was therefore surprising that PGHS-1-deficient mice had a $99 \%$ reduction in gastric $\mathrm{PGE}_{2}$ levels but did not spontaneously develop ulcers (1). Initially, many researchers attributed this absence of gastric ulceration in PGHS-1-null mice to compensatory mechanisms. However, more recently, a novel PGHSdependent mechanism of NSAID-induced gastric ulceration has been reported (17). In these studies, neither the PGHS-1-selective inhibitor SC-560 nor the PGHS-2-selective inhibitor celecoxib sufficed to induce ulcers, but gastric damage occurred when the two NSAIDs were administered in combination. These studies may explain why PGHS-1-deficient mice do not spontaneously develop ulcers. Presumably, if inhibition of PGHS activity is the sole mechanism of NSAIDinduced ulceration, administering PGHS-1 inhibitors to PGHS-2-null mice or PGHS-2 inhibitors to PGHS-1-null mice would be ulcerogenic.

In addition to its role in resolving inflammation $(15,16)$, PGHS-2 has been shown to promote ulcer healing. Indomethacin-induced ulcers heal poorly in PGHS-2-null mice compared with wild-type mice and ultimately prove fatal (15). Like the PGHS-2-deficient mice, PGHS- 2 selective NSAIDs prolong the healing of ulcers in rats and mice $(18,19)$. Thus, as in inflammation, there are roles for PGHS-2 in both the onset and resolution of a disease state, a matter that may be relevant when considering chronic use of PGHS-2-selective drugs.

Carcinogenesis. To date, most studies have implicated PGHS-2, rather than PGHS-1, as the isoform involved in colon carcinogenesis. In a recent study, however, we determined that deficiency for either PGHS-1 or PGHS-2 reduces polyp formation in $\mathrm{Min}^{-/+}$ mice about equally ( $\sim 80 \%$; ref. 20$)$. These studies raise the question of whether PGHS-1 and PGHS- 2 contribute to tumorigenesis through common or different mechanisms. Only a speculative answer to this question can be given at present. Based on recent work by Houchen et al. (21), it is possible that PGHS- 1 functions in early stages of tumorigenesis by protecting initiated stem cells from DNA damage-induced death, whereas PGHS- 2 contributes to tumor promotion after loss of heterozygosity of the APC gene has occurred (22). Studies by Chulada et al. (20) indicate that at the adenoma stage of tumor development, PGHS-2 is present in the interstitial cells rather than the epithelial cells of the tumor, and thus, that prostanoids formed via PGHS-2 exert a paracrine rather than an autocrine effect to promote tumor growth. Work with PGHS null mice indicates that both isozymes contribute to the increased $\mathrm{PGE}_{2}$ production seen in polyps. Overall, these results suggest that both PGHS-1 and PGHS-2 play key roles in intestinal tumorigenesis and that PGHS-1 may also be an effective chemotherapeutic target for NSAIDs.

\section{Biochemical evidence for unique PGHS-1}

and PGHS-2 biosynthetic pathways

For there to be isozyme-specific effects, PGHS-1 and PGHS-2 must be able to function independently at the biochemical level. This can be achieved simply by segregating the isozymes into different cells. However, in many instances PGHS- 1 and PGHS- 2 are coexpressed in the same cells. Under the latter conditions, the independent functioning of PGHS isozymes appears to involve metabolic rather than physical segregation of these enzymes. The two PGHS isozymes are found predominately in the same organelles, at the lumenal surfaces of the endoplasmic reticulum and nuclear envelope of cells (23), although it is conceivable that each isoform is localized to a specific subdomain of these membranes. Neither enzyme appears to interact directly with $c \mathrm{PLA}_{2}$ or $\mathrm{SPLA}_{2} \mathrm{~s}$, the phospholipases that mobilize AA. It is possible that PGHS-1 or PGHS- 2 can bind to the downstream synthases, such as $\mathrm{TXA}_{2}$ synthase, $\mathrm{PGI}_{2}$ synthase, and/or microsomal (m) PGE synthase (all of which enzymes are associated with the endoplasmic reticulum; refs. 24, 25); however, to date, there is no evidence for such interactions.

\section{PGHS-2-independent functions of PGHS-1}

When PGHS-1 and PGHS- 2 are both expressed in the same cells, supplying AA either exogenously or via activation of cellular lipases always leads to the functioning of PGHS-2. Only when PGHS-2 is absent does PGHS-1 appear to function independently; but because PGHS- 1 is a constitutive enzyme, whereas PGHS-2 is inducible, this is actually the usual situation. PGHS-2 is generally present in cells only during early stages of cell differentiation or replication (25). There is considerable literature on the regulation of PGHS-2 gene expression, but there is little information about how the expression of PGHS- 1 is controlled (25).

\section{PGHS-1-independent functions of PGHS-2}

In vitro at high substrate/enzyme ratios, PGHS-1 and PGHS-2 have quite similar kinetic properties including their $\mathrm{K}_{\mathrm{m}}$ values for AA $(\sim 5 \mu \mathrm{M}$; ref. 25$)$. However, the $\mathrm{K}_{\mathrm{m}}$ value of PGHS-1 for AA appears to be higher than that of PGHS-2 when the enzymes are studied in cultured cells $(\sim 2 \mu \mathrm{M}$ and $10 \mu \mathrm{M}$, respectively; refs. 26,27$)$. Thus, in intact cells expressing both isoforms, PGHS- 2 can function in the presence of a nonfunctioning PGHS-1 
if the AA concentrations are kept relatively low. This mechanism may account for the fact that prostanoids formed in the so-called "late phase," a period defined as occurring 2-6 hours after treating cells with tumor promoters, cytokines, or growth factors, are formed primarily via PGHS-2.

The intracellular concentration of free AA depends on competing actions of various lipases, which mobilize arachidonate from phosphoglycerides, and CoA ligases, which scavenge free fatty acids. There is a consensus that $\mathrm{CPLA}_{2}$ is involved in mobilizing AA for PGHS- 1 but that prostaglandin formation during the late phase and involving PGHS-2 depends on both $\mathrm{CPLA}_{2}$ and one or more $\mathrm{SPLA}_{2} \mathrm{~s}$ (see Fitzpatrick and Soberman, this Perspective series, ref. 28; see also refs. 27 and 29-31).

The basis of the apparent $K_{\mathrm{m}}$ differences between PGHS-1 and PGHS- 2 in intact cells is not clear. However, this situation can be mimicked in vitro with purified PGHSs when measuring rates of product formation at low concentrations of both AA and lipid hydroperoxide. The biochemical explanation for this rather complex type of regulation is as follows: PGHSs catalyze both a cyclooxygenase reaction, in which AA plus two $\mathrm{O}_{2}$ molecules are converted to $\mathrm{PGG}_{2}$, and a peroxidase reaction, in which $\mathrm{PGG}_{2}$ is reduced to $\mathrm{PGH}_{2}$ (ref. 25; see also Serhan and Oliw, this Perspective series, ref. 32). For the cyclooxygenase activities of PGHS-1 and PGHS- 2 to function, the heme group at the peroxidase site must undergo a lipid peroxide-dependent oxidation. The oxidized heme then oxidizes a tyrosine in the cyclooxygenase site that, in turn, abstracts a hydrogen atom from $\mathrm{AA}$, yielding an AA radical that reacts with $\mathrm{O}_{2}$ to form $\mathrm{PGG}_{2}(25)$ (itself a lipid peroxide). The concentration of lipid peroxide required to activate the cyclooxygenase activity of PGHS-1 is about ten times higher than that necessary for PGHS-2 in the presence of low AA concentrations (33). Thus, a combination of (a) low endogenous peroxide concentrations within the lumen of the endoplasmic reticulum, where PGHS-1 and PGHS- 2 are predominately localized in intact cells (23) and (b) AA concentrations below $2 \mu \mathrm{M}$ (i.e., at relatively high enzyme/substrate ratios; refs. 33,34 ) would favor the operation of the cyclooxygenase activity of PGHS-2 over that of PGHS-1. Regardless of the mechanism, it appears that the cyclooxygenase activities of PGHS- 1 and PGHS- 2 are controlled differentially by regulating the amount of lipid peroxide and AA available to the enzymes.

Alternative substrates for PGHS-2. There are subtle differences in the fatty acid substrate specificities between PGHS-1 and PGHS-2 (25), but these are probably of little biologic significance beyond ensuring that both enzymes act on AA in preference to other fatty acids substrates. However, PGHS-2 but not PGHS-1 can use esterified fatty acid substrates (35). One example is 2arachidonyl glycerol, which can be generated from phosphatidylinositol by the sequential actions of phospholipase C and diglyceride lipase (35). 2-Arachidonyl glycerol is formed by a mouse macrophage line and converted to 2-PGD 2 glycerol via PGHS-2 and PGD synthase (35). This provocative observation raises a host of questions regarding the relative importance of the classical and alternative pathways. For example, it is possible that AA is normally not even the physiologically relevant substrate for PGHS-2. Considerable further work needs to be done on this topic, including determining whether 2- $\mathrm{PGH}_{2}$ glycerol can be converted to 2-prostanoid glycerols other than 2-PGD 2 glycerol (e.g., 2-PGI 2 glycerol).

Coupling of PGHS-1 and PGHS-2 to downstream synthases. A final way in which PGHS-1 and PGHS-2 biosynthetic pathways may be dissociated metabolically is by a preferential coupling of the isoforms to various downstream synthases (see Fitzpatrick and Soberman, this Perspective series, ref. 28). PGHS-2 appears to be coupled metabolically to $\mathrm{PGI}_{2}$ formation in the uterus in association with decidualization and implantation (12). There also appears to be an mPGE synthase that is coordinately induced with PGHS-2 and seems to function preferentially with PGHS-2, rather than PGHS-1 $(24,36)$.

\section{Concluding remarks}

The data obtained with PGHS-1 and PGHS-2 deficient mice clearly show differences in some physiological actions of the two isoforms, and some observed physiological roles are quite different from those originally expected. For example, it was quite surprising that mice deficient in PGHS-1, the constitutive isoform believed to be responsible for homeostasis, are healthy and live a normal life span, whereas mice lacking PGHS-2 expression, the inducible isoform thought to be expressed primarily in disease states, display various developmental problems, female reproductive disorders, and a shortened life span. Most recent studies have demonstrated a concordance between the physiological effects of the genetic deficiency of the PGHS isoforms and isoform-specific or -nonspecific effects of NSAIDs. However, both the drug studies and the studies of PGHS deficient mice have their limitations. Whereas NSAIDs may cause some of their effects by mechanisms other than PGHS inhibition, compensation mechanisms or developmental changes may influence the responses of PGHS-deficient mice. Inflammation, for example, cannot be fully elucidated with PGHS-2-deficient mice, as PGHS-2 acts at two different steps in this process, initiation and resolution. Other prostaglandin-regulated responses may also require PGHSs to function at multiple steps. On the other hand, PGHS-null mice probably do model the development of certain diseases caused by PGHS inhibitors. Furthermore, in the PGHS-null mice there are examples of the remaining isoform being upregulated and compensating for the missing isoform; further studies will be needed to deter- 
mine whether such upregulation contributes to the normal physiological process as it occurs in wild-type animals. Future biochemical studies will likely focus on the mechanisms by which different lipases and downstream synthases are coupled physically or metabolically to PGHS- 1 and/or PGHS-2, why the $K_{\mathrm{m}}$ values for PGHS-1 and PGHS- 2 are different in intact cells, and whether AA, 2-arachidonyl glycerol or another lipid is the preferred substrate for PGHS-2.

\section{Acknowledgments}

Work conducted in the laboratory of W.L. Smith and described in this report was supported in part by grants from the NIH (DK22042 and PO1 GM57323).

1. Langenbach, R., et al. 1995. Prostaglandin synthase 1 gene disruption in mice reduces arachidonic acid-induced inflammation and indomethacin-induced gastric ulceration. Cell. 83:483-492.

2. Morham, S.G., et al. 1995. Prostaglandin synthase 2 gene disruption causes severe renal pathology in the mouse. Cell. 83:473-482.

3. Dinchuk, J.E., et al. 1995. Renal abnormalities and an altered inflammatory response in mice lacking cyclooxygenase II. Nature. 378:406-409.

4. Brash, A.R. 2001. Arachidonic acid as a bioactive molecule. J. Clin. Invest. 107:1339-1345.

5. Sugimoto, Y., Narumiya, S., and Ichikawa, A. 2000. Distribution and function of prostanoid receptors: studies from knockout mice. Prog. Lipid Res. 39:289-314.

6. Patrignani, P., et al. 1994. Biochemical and pharmacological characterization of the cyclooxygenase activity of human blood prostaglandin endoperoxide synthases. J. Pharmacol. Exp. Ther. 271:1705-1712.

7. Patrono, C. 1998. Prevention of myocardial infarction and stroke by aspirin: different mechanisms? Different dosage? Thromb. Res. 92(Suppl.):S7-S12.

8. Lim, H., et al. 1997. Multiple female reproductive failures in cyclooxygenase 2-deficient mice. Cell. 91:197-208.

9. Gross, G.A., et al. 1998. Opposing actions of prostaglandins and oxytocin determine the onset of murine labor. Proc. Natl. Acad. Sci. USA 95:11875-11879.

10. Gross, G., et al. 2000. Inhibition of cyclooxygenase-2 prevents inflammation-mediated preterm labor in the mouse. Am. J. Physiol. Regul. Integr. Comp. Physiol. 278:R1415-R1423.

11. Davis, B.J., et al. 1999. Anovulation in cyclooxygenase-2-deficient mice is restored by prostaglandin E2 and interleukin-1beta. Endocrinology. 140:2685-2695.

12. Lim, H., et al. 1999. Cyclo-oxygenase-2-derived prostacyclin mediates embryo implantation in the mouse via PPARdelta. Genes Dev. 13:1561-1574.

13. Komhoff, M., et al. 2000. Cyclooxygenase-2-selective inhibitors impair glomerulogenesis and renal cortical development. Kidney Int. 57:414-422

14. Loftin, C.D., et al. 2001. Failure of ductus arteriosus closure and remodeling in neonatal mice deficient in cyclooxygenase-1 and -2. Proc. Natl. Acad. Sci. USA. 98:1059-1064.

15. Wallace, J.L., et al. 1998. Cyclooxygenase 1 contributes to inflammatory responses in rats and mice: implications for gastrointestinal toxicity. Gastroenterology. 115:101-109.

16. Gilroy, D.W., et al. 1999. Inducible cyclooxygenase may have antiinflammatory properties. Nat. Med. 5:698-701.

17. Wallace, J.L., McKnight, W., Reuter, B.K., and Vergnolle, N. 2000. NSAID induced gastric damage in rats: requirement for inhibition of both cyclooxygenase 1 and 2. Gastroenterology. 119:706-714.
18. Mizuno, H., et al. 1997. Induction of cyclooxygenase 2 in gastric mucosal lesions and its inhibition by the specific antagonist delays healing in mice. Gastroenterology. 112:387-397.

19. Shigeta, J., Takahashi, S., and Okabe, S. 1998. Role of cyclooxygenase-2 in the healing of gastric ulcers in rats. J. Pharmacol. Exp. Ther. 286:1383-1390.

20. Chulada, P.C., et al. 2000. Genetic disruption of Ptgs-1, as well as Ptgs-2, reduces intestinal tumorigenesis in Min mice. Cancer Res. 60:4705-4708.

21. Houchen, C.W., Stenson, W.F., and Cohn, S.M. 2000. Disruption of the cyclooxygenase- 1 gene results in an impaired response to radiation injury. Am. J. Pathol. 279:G858-G865.

22. Prescott, S.M., and White, R.L. 1996. Self-promotion? Intimate connections between APC and prostaglandin H synthase-2. Cell. 87:783-786.

23. Spencer, A.G., Woods, J.W., Arakawa, T., Singer, I.I., and Smith, W.L. 1998. Subcellular localization of prostaglandin endoperoxide $\mathrm{H}$ synthases- 1 and -2 by immunoelectron microscopy. J. Biol. Chem. 273:9886-9893.

24. Murakami, M., et al. 2000. Regulation of prostaglandin E2 biosynthesis by inducible membrane-associated prostaglandin E2 synthase that acts in concert with cyclooxygenase-2. J. Biol. Chem. 275:32783-32792.

25. Smith, W.L., DeWitt, D.L., and Garavito, R.M. 2000. Cyclooxygenases: structural, cellular and molecular biology. Annu. Rev. Biochem. 69:149-182.

26. Shitashige, M., Morita, I., and Murota, S. 1998. Different substrate utilization between prostaglandin endoperoxide $H$ synthase- 1 and -2 in NIH3T3 fibroblasts. Biochemica et Biophysica Acta. 1389:57-66.

27. Murakami, M., Kambe, T., Shimbara, S., and Kudo, I. 1999. Functional coupling between various phospholipase A2s and cyclooxygenases in immediate and delayed prostanoid biosynthetic pathways. J. Biol. Chem. 274:3103-3115.

28. Fitzpatrick, F.A., and Soberman, R. 2001. Regulated formation of eicosanoids. J. Clin. Invest. 107:1347-1351.

29. Bezzine, S., et al. 2000. Exogenously added human group X secreted phospholipase $\mathrm{A}(2)$ but not the group IB, IIA, and V enzymes efficiently release arachidonic acid from adherent mammalian cells. J. Biol. Chem. 275:3179-3191.

30. Gijon, M.A., Spencer, D.M., Siddiqi, A.R., Bonventre, J.V., and Leslie, C.C. 2000. Cytosolic phospholipase A2 is required for macrophage arachidonic acid release by agonists that do and do not mobilize calcium. Novel role of mitogen-activated protein kinase pathways in cytosolic phospholipase A2 regulation. J. Biol. Chem. 275:20146-20156.

31. Balsinde, J., Balboa, M.A., and Dennis, E.A. 2000. Identification of a third pathway for arachidonic acid mobilization and prostaglandin production in activated P388D1 macrophage-like cells. J. Biol. Chem. 275:22544-22549.

32. Serhan, C.N., and Oliw, E. 2001. Unorthodox routes to prostanoid formation: new twists in cyclooxygenase-initiated pathways. J. Clin. Invest. 107:1481-1489.

33. Chen, W., Pawelek, T.R., and Kulmacz, R.J. 1999. Hydroperoxide dependence and cooperative cyclooxygenase kinetics in prostaglandin $\mathrm{H}$ synthase-1 and -2. J. Biol. Chem. 274:20301-20306.

34. Swinney, D.C., Mak, A.Y., Barnett, J., and Ramesha, C.S. 1997. Differential allosteric regulation of prostaglandin $H$ synthase 1 and 2 by arachidonic acid. J. Biol. Chem. 272:12393-12398.

35. Kozak, K.R., Rowlinson, S.W., and Marnett, L.J. 2000. Oxygenation of the endocannabinoid, 2-arachidonylglycerol, to glyceryl prostaglandins by cyclooxygenase-2. J. Biol. Chem. 275:33744-33749.

36. Tanioka, T., Nakatani, Y., Semmyo, N., Murakami, M., and Kudo, I. 2000. Molecular identification of cytosolic prostaglandin E2 synthase that is functionally coupled with cyclooxygenase- 1 in immedite prostaglandin E2 biosynthesis. J. Biol. Chem. 275:32775-32782.

37. Rocca, B., et al. 1999. Distinct roles of prostaglandin $\mathrm{H}$ synthases 1 and 2 in T-cell development. J. Clin. Invest. 103:1469-1477.

38. Riehl, T., Cohn, S., Tessner, T., Schloemann, S., and Stenson, W.F. 2000. Lipopolysaccharide is radioprotective in the mouse intestine through a prostaglandin-mediated mechanism. Gastroenterology. 118:1106-1116. 